\title{
Multiscale Analysis of the Strength Deterioration of Loess under the Action of Drying and Wetting Cycles
}

\author{
Yang Bai iD, Wanjun Ye, Yuntao Wu, and Yiqian Chen \\ School of Architecture and Civil Engineering, Xi'an University of Science and Technology, Xi'an 710054, Shaanxi, China \\ Correspondence should be addressed to Yang Bai; baiyang19920613@163.com
}

Received 1 February 2021; Revised 2 March 2021; Accepted 25 March 2021; Published 2 April 2021

Academic Editor: Hamed Akhavan

Copyright (c) 2021 Yang Bai et al. This is an open access article distributed under the Creative Commons Attribution License, which permits unrestricted use, distribution, and reproduction in any medium, provided the original work is properly cited.

\begin{abstract}
To study the strength degradation mechanism of compacted loess during dry-wet cycles, $0-5$ dry-wet cycles tests and many triaxial compression tests were carried out on loess with an optimal moisture content. During the dry-wet cycles, the loess samples were analyzed by nuclear magnetic resonance and scanning electron microscopy. Studies have shown that at the macro level, with increasing numbers of wet and dry cycles and increasing cycle amplitude, the cohesive force and internal friction angle of the loess decrease, and the shear strength of the loess deteriorates significantly. At the micro level, with the number of wet and dry cycles increasing, the connection between particles changes from surface-to-surface contacts to point-to-point or point-to-surface contacts. The edges and corners of the particles decrease, the roundness increases, the large pores gradually decrease, the small pores gradually increase, and the fractal dimension gradually increases. In terms of microscopic view, the NMR test shows that with increasing numbers of dry-wet cycles, the T2 peak curve increases, the curve width increases slightly, the peak area gradually increases, and the porosity increases. From the macroscopic, mesoscopic, and microscopic multiscale analysis, the structure of loess is degraded under the action of dry and wet cycles; the strength of the loess is degraded significantly after 0 to 3 cycles and then gradually stabilizes. These research results can provide a certain reference value for the management of loess collapse geological disasters in semiarid climates.
\end{abstract}

\section{Introduction}

In Northwest China, loess is distributed everywhere. However, due to the complex terrain, vertical and horizontal ravines, harsh environment, and unique climate in the loess area, and under natural and anthropogenic actions, loess is extremely prone to disasters such as landslides, collapses, and soil erosion, which seriously endanger engineering construction and the safety of people's lives and property and restrict the sustainable development of the local economy $[1,2]$. This article focuses on water, a key factor that causes loess collapse, and studies loess bodies through the evaporation of water, that is, dry-wet cycles; the results reveal the mechanism of loess structural degradation from a multiscale perspective. The study has important academic value, scientific significance, and essential practical value and significance for the construction of basic projects in the future. The research results will also provide a more scientific theoretical basis for the engineering practice of disaster prevention and mitigation in the process of project site selection, construction, and operation in the loess area.

Many scholars have conducted various studies on the effects of dry-wet cycles on soil properties. Ma et al. [3] performed X-ray microcomputer tomography on shale and Quaternary red clay using 3D microtomography imaging technology to analyze the pore structure changes in the samples at depth under the action of dry-wet cycles. Razouki et al. [4] conducted a systematic experimental study on the strength and deformation of gypsum-rich sand used in subgrade construction under the action of dry-wet cycles and obtained certain results. Pasculli et al. [5] observed the significant influence of dry-wet cycles on the crushing of pumice and the resulting structural collapse during laboratory tests. Wu et al. [6] studied the mechanical properties of improved expansive soil under the action of dry-wet cycles and provided a reference for the construction of 
roadbeds and other projects. de Oliveira et al. [7] used X-ray computed tomography to study the porosity changes in cultivated soil under the action of dry-wet cycles; Liu et al. [8] used triaxial strength tests, nuclear magnetic resonance, and scanning electron microscopy on expansive soil under the action of dry-wet cycles to study the macromechanical behavior and the microstructure damage to the regularity of soil.

For studying loess, the current research mainly focuses on the mechanical properties of loess under dry-wet and loading cycles. For example, Ye et al. [9] initially explored the influence of water migration on the strength of compacted loess and its mechanism under the action of dryness and wetness in seasonal frozen soil regions; Chou et al. [10] analyzed the influence of structure, dryness and wetness, initial moisture content, wet weight, and load size on the collapsibility coefficient of different structural loess under the action of dryness and wetness; Su et al. [11] used scanning electron microscopy (SEM) to quantitatively analyze the swelling and shrinking deformation characteristics of undisturbed loess under the action of wet and dry cycles and the evolution of cracks; $\mathrm{Hu}$ et al. [12] carried out a dry-wet cycle triaxial test considering the three relevant factors of dry density, drywet cycle amplitude, and lower limit water content of a dry-wet cycle and obtained compacted loess strength degradation curves under the influence of the different factors; Li et al. [13] conducted a laboratory study on undisturbed loess and used direct shear tests to analyze the changes in shear strength of undisturbed loess under dry-wet conditions. The results showed that the mechanical properties of loess under the two conditions greatly deteriorated; Wen Shaojie, Zhang Zelin, and Zhang Jun [14-16] studied the mechanical properties of loess under dynamic action and obtained useful results.

The actions of water and soil also have certain influences on the stability of slopes. Tang [17] studied the effect of chemical damage by water on the elastic modulus of rock and found that the influences of the chemical action of an aqueous solution and the rock soil on the elastic modulus of rock are closely related. Wang [18] analyzed the regular change in the shear strength of the soil under the action of water-soil chemistry and obtained the impact of the ion concentrations in the aqueous solution on the original viscosity of the soil under the action of water. Cohesion and inherent cohesion have significant weakening effects but have little effect on the angle of internal friction. Through research on the physical and mechanical properties of clay under variations in the water chemical environment, Yan [19] showed that the variation in the water chemical environment has an important influence on the mechanical properties of clay and proposed to establish a theoretical framework of soil mechanics based on the coupling of chemistry and mechanics. In summary, the basic mechanical properties of loess have been studied extensively, and many conclusions have been obtained. The author has also conducted a study on the degradation of the internal structure of loess under the action of dry-wet cycles [20]. However, there is no deep research on the changes in the pore structure of loess from the mesostructure. Based on the macroscopic triaxial test, the author used electron scanning microscopy (SEM) and nuclear magnetic resonance (NMR) technology to analyze the pore structure of loess under different cycles and conducted a multiscale analysis of the changes in the mechanical properties of loess under the action of dry-wet cycles from different perspectives. This study aims to provide a reference for the management of loess collapse geological disasters in semiarid areas.

\section{Experimental Procedure}

2.1. Experimental Material. In this article, a landslide loess from Yan'an was used to provide soil samples for laboratory experiments. The sample taken is the S1 loess from the middle Pleistocene (Q3) loess sequence. To analyze the physical properties of the soil samples, the soil samples taken were subjected to laboratory geotechnical experiments, and the basic physical properties of the soil samples were obtained as shown in Table 1.

2.2. Experimental Process. The test process is shown in Figure 1. The main test equipment included (a) a negative pressure vacuum saturation instrument, (b) an ultrasonic testing analyzer, (c) a standard unsaturated soil triaxial test system produced by the British GDS company, (d) a nuclear magnetic resonance instrument, and (e) a scanning electron microscope.

2.3. Test Plan and Principle. The test plan is designed based on loess from the Luochuan Plateau to study the changes in grain pores and test the degradation of loess strength under repeated dry-wet cycles.

2.3.1. Dry-Wet Cycle Test. According to on-site soil sampling, indoor water content measurement, and data collection, the water content varies greatly $\left(\omega_{\max }=25 \%\right.$ and $\omega_{\min }=8 \%$ ). Therefore, to study the changes in the physical and mechanical properties of loess under the action of dry-wet cycles, dry-wet cycle tests were carried out with different numbers and amplitudes of cycles. The undisturbed soil sample was taken from the site to the laboratory, and the dripping method and natural air drying method were used to change the moisture content of the undisturbed soil; a moisture content of $16 \%$ was selected as the control point for the dry-wet cycle tests, and $0,1,2,3,4$, and 5 dry-wet cycles were conducted and tested. The quality of the control sample was used to determine the changes in moisture content. When the moisture content reached the control point, a triaxial consolidation undrained shear test was performed. To expedite the test process, an electric constant-temperature heating and drying oven was added to dry the soil samples; the prepared soil samples were placed with initial water content in the temperature-controlled oven, and the dripping method was then used to evenly moisturize the samples at room temperature before 
TABLE 1: Test statistics of various physical and mechanical parameters of Yan'an loess.

\begin{tabular}{|c|c|c|c|c|c|c|c|c|}
\hline Statistics & $\begin{array}{c}\text { Moisture } \\
\text { content } w(\%)\end{array}$ & $\begin{array}{c}\text { Natural density } \\
\rho\left(\mathrm{g} / \mathrm{cm}^{3}\right)\end{array}$ & Void ratio $e$ & $\begin{array}{c}\text { Particles } \\
\text { proportion } G_{s}\end{array}$ & $\begin{array}{l}\text { Dryness density } \\
\rho\left(\mathrm{g} / \mathrm{cm}^{3}\right)\end{array}$ & $\begin{array}{l}\text { Liquid } \\
\operatorname{limit} w_{L}\end{array}$ & $\begin{array}{l}\text { Plastic } \\
\operatorname{limit} w_{P}\end{array}$ & $\begin{array}{l}\text { Plastic } \\
\text { index } I_{P}\end{array}$ \\
\hline Max & 22.75 & 1.84 & 1.28 & 2.73 & 1.58 & 33.4 & 21.7 & 10.8 \\
\hline Min & 12.81 & 1.42 & 0.76 & 2.71 & 1.21 & 26.4 & 18.6 & 8.5 \\
\hline Average & 16.5 & 1.58 & 0.96 & 2.72 & 1.38 & 30.26 & 20.61 & 9.45 \\
\hline
\end{tabular}

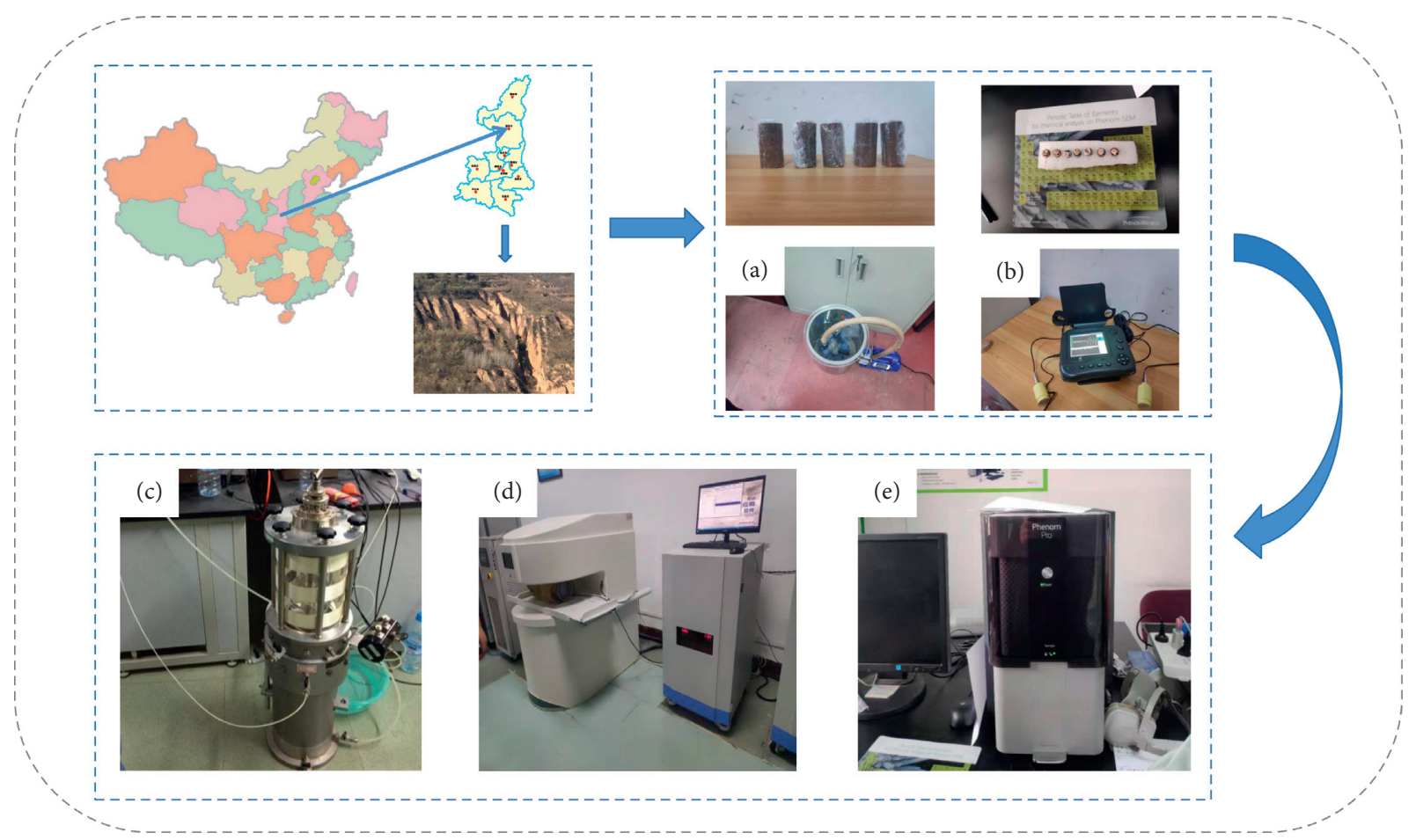

FIgURE 1: Test process diagram.

drying. This was the first dry-wet cycle; the process was then repeated to obtain the third and fifth dry-wet cycle soil samples. The schematic diagram of the dry and wet cycle process is shown in Figure 2.

2.3.2. Loess Strength Degradation Tests. To fully investigate the effect of dry and wet cycles on the strength degradation of compacted loess, 4 types of dry and wet cycle paths were set up; the test numbers were a, $b, \mathrm{c}$, and $d$, and the lower limit moisture contents were set to $17.8 \%, 12.8 \%, 7.9 \%$, and $2.9 \%$, respectively; the corresponding dry-wet cycle amplitudes $(A)$ were $6.2 \%, 11.2 \%, 16.2 \%$, and $21.2 \%$. The aim of this procedure was to simulate the cyclical process of saturation at different depths (rising water level), dehumidification (falling water level), resaturation, and further dehumidification after the completion of compacted loess construction. For the saturation of the sample, the vacuum saturation method was adopted. The moisture content after the test was approximately $25 \%$, the difference did not exceed $0.1 \%$, and the saturation was more than $98 \%$; the drying method $\left(40^{\circ} \mathrm{C}\right)$ was used for the dehumidification process.
According to the test, multiple sets of triaxial undisturbed soil samples were prepared by the water distribution method to obtain samples with optimal moisture content. Using conventional triaxial tests, each group of soil samples was subjected to dry-wet cycle tests with different cycle numbers and amplitudes under the conditions with confining pressures $\sigma_{3}=100 \mathrm{kPa}, 200 \mathrm{kPa}$, and $300 \mathrm{kPa}$ and a shear rate of $0.2 \mathrm{~mm} / \mathrm{min}$ to study the strength and dry-wet cycles of unsaturated loess. The experiments adopted the method of the consolidated undrained (CU) shear test. The GDS triaxial instrument was used to perform triaxial tests on the Luochuan Plateau loess. The dimensions of the conventional triaxial samples were diameter $=3.91 \mathrm{~cm}$ and height $H=8.0 \mathrm{~cm}$. To study the regularity of the strength degradation of loess under the action of dry-wet cycles, the stress-strain curve of unsaturated loess for dry-wet cycles was studied under the condition of controlled confining pressure.

2.3.3. Scanning Electron Microscope Tests. The scanning electron microscope tests used the Phenom Pro desktop scanning electron microscope made by Holland Feiner; the 
maximum total magnification was 130,000 times, and the vacuum time was less than 1 minute.

The soil samples were scanned after different dry-wet cycles from the initial moisture content, and approximately $1-3 \mathrm{~cm}$ was selected after the dry-wet cycles. The samples were fully dried before scanning, and the surface was coated with gold to better reflect the signal.

2.3.4. NMR Tests. The nuclear magnetic resonance tests used the NMRC12-010V nuclear magnetic resonance pore analyzer introduced by the School of Architecture and Civil Engineering of Xi'an University of Science and Technology. According to the principle of low-field NMR, the hydrogen nucleus is regarded as a good signal carrier. Under the action of an external magnetic field, the hydrogen nucleus can be considered a small magnetic needle. The torque generated by the external magnetic field aligns the spin axis of the hydrogen nucleus with the direction of the magnetic field. The nuclei in the magnetic field are arranged in one direction. According to quantum mechanics theory, when a hydrogen nucleus is in an external magnetic field, protons are decomposed into two energy states: high energy and low energy. The difference in energy states means that the number of spins parallel to the external magnetic field is slightly greater than the number of nuclear spins opposite to the external magnetic field. The difference between the two numbers forms the magnetization vector M0, which provides a measurement signal for NMR. Usually, we use NMR relaxation measurements to express the porosity, pore size distribution, and other information of a porous medium.

The relationship between the pore size in the loess and the relaxation time $\mathrm{T} 2$ is obtained:

$$
\frac{1}{T_{2}}=\rho_{2}\left(\frac{S}{V}\right)_{\text {Porosity }} .
$$

The saturated sample that had undergone the dry-wet cycle together with the PVC pipe was placed into the NMR room for testing, and the test parameters were adjusted. The specific parameters were as follows: RF delay $\mathrm{RFD}=0.002 \mathrm{~ms}$; analog gain $\mathrm{GF} 1=20 \mathrm{~dB}$; digital gain DRG1 $=3 ; 90^{\circ}$ pulse width $P 1=34 \mathrm{~s} ; 180^{\circ}$ pulse width $P 2=68$ s; preamplification multiple $P R G=1$; waiting time $\mathrm{TW}=2000 \mathrm{~ms}$; echo time $\mathrm{TE}=0.21 \mathrm{~ms}$; number of echoes $\mathrm{NGCH}=2000$; accumulation times $\mathrm{NS}=16$; main frequency $s f=12 \mathrm{MHz}$; frequency drift ol $=479043.8 \mathrm{~Hz}$.

\section{Analysis of Test Results}

3.1. Stress-Strain Relationship. The triaxial shear stressstrain relationship curves for compacted loess with different dry-wet cycles and different dry-wet cycle paths are shown in Figure 3. Due to space limitations, only the stress-strain curve of each dry-wet cycle path at a confining pressure of $100 \mathrm{kPa}$ is listed here, which represents the stress value of the undisturbed loess at a certain strain.

The figure shows that under the action of wet and dry cycles, with increasing numbers of wet-dry cycles, the stressstrain curve evolves to having no peak point, the shape of the stress-strain curve can be changed from weak softening to hardening, and the amplitude of the hardening curve becomes increasingly obvious. The position of the strain curve is lowered, and the variation range of the principal stress difference shows a decreasing trend, indicating that the loess strength is gradually deteriorating; a comparative analysis of the compacted loess stress-strain curves for different dry-wet cycle paths can be obtained. As the number of dry-wet cycles increases, the stress-strain curve of each dry and wet cycle path moves toward the bottom. Under the same number of cycles, the greater the cycle amplitude is, the lower the shear strength.

\subsection{Triaxial Shear Strength of Compacted Loess under Dry Wet} Cycles. To further analyze the changes in the triaxial shear strength of compacted loess under the action of dry-wet cycles, the triaxial shear strength of each cycle path for different numbers of dry-wet cycles is statistically analyzed, and the triaxial shear strength and the dry-wet cycles are established. Their relationship " $n$ " is shown in Figure 4.

Figure 4 illustrates that, for the same number of dry-wet cycles, as the confining pressure increases, the shear strength of the soil gradually increases. Under the same confining pressure, with increasing numbers of initial cycles, the shear strength of the soil gradually decreases. Another phenomenon is clearly observed in the figure. The shear strength decreases the most during a dry-wet cycle. When $\sigma 3=100 \mathrm{kPa}$ and the dry-wet cycle path is "a", after the first dry-wet cycle, the soil shear strength decreases by $11.3 \%$. When the dry-wet cycle path is " $b$ ", the soil shear strength decreases by $31.7 \%$. When the dry-wet cycle path is " $c$ ", the soil shear strength decreases by $40.2 \%$. When the dry-wet cycle path is " $d$ ", the shear strength of the soil decreases by $51.0 \%$; from the 2 nd cycle to the 5 th cycle, the shear strength tends to be stable.

The figure also shows that the triaxial shear strength of the sample soil is significantly affected by the dry-wet cycle path (dry-wet cycle amplitude). The triaxial shear strength of different circulation paths increases with the number of drywet cycles, and its slope of the curve gradually decreases. Before 3 cycles, the decrease is greater; when the number of cycles is 1 , the curve is the steepest, and the slope is the largest. After 3 cycles, the curve tends to become stable. Comparing and analyzing the different dry-wet cycle paths under the same confining pressure and the intensity value corresponding to the lowest point of the relationship curve for the number of dry-wet cycles, it can be determined that the intensity value of dry-wet cycle path " $d$ " is the smallest, followed by path "c" and path "b", and path "a" has the largest intensity value.

According to the Mohr-Coulomb theory, the shear strength index, cohesion $c$, and internal friction angle $\varphi$ can be obtained. The relationship between the cohesive force $c$, the internal friction angle $\varphi$, and the number of dry-wet cycles $n$ is shown in Figures 5 and 6. Figure 5 indicates that the cohesive force of compacted loess decreases sharply and then gradually increases with the increase in the number of dry-wet cycles, and the curve has an obvious turning point; 


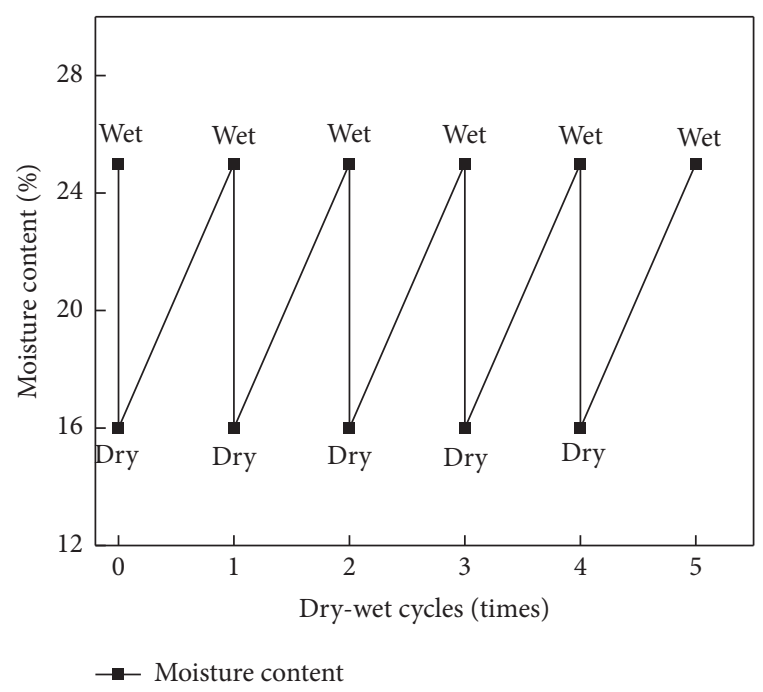

FIgURE 2: Schematic diagram of the dry and wet cycle process.

the relationship between the turning point and the number of wet and dry cycles is evident. The curve before the turning point is steeper and has a larger slope than the curve beyond the turning point. The cohesion and internal friction angle change greatly with 0 to 3 cycles. After 3 dry and wet cycles, the cohesion forces for pathways "a", "b", "c", and "d" decrease by $14.19 \mathrm{kPa}, 30.14 \mathrm{kPa}, 65.2 \mathrm{kPa}$, and 79.66 , respectively. The slope of the curve between the cohesive force and internal friction angle of loess and the number of drywet cycles is gradually reduced, and the slope of the curve slowly becomes flat after 3 cycles.

The main reason for the deterioration of the cohesive force is that the adsorption strength continuously decreases with the dry-wet cycles, and the adsorption strength is closely related to the pore characteristics of the soil. From a mesoscopic point of view, the matrix suction in the soil is caused by the surface tension of the liquid bridges. During the dehumidification process, the moisture content of the soil decreases, and the volume of the liquid bridges connecting the soil particles decreases. The volume of the liquid bridges connecting the soil particles decreases, and the matrix suction becomes larger. When the soil is cracked by the critical matrix suction, the liquid bridges between the soil particles are broken, resulting in cracks in the soil [21]. An increase in the cyclic amplitude prolongs the cracking process, resulting in an increase in the degrading effect of cohesion. When the water content is relatively low, the suction force of the matrix is very large, and as the water content decreases, the possibility of liquid bridge rupture gradually increases, resulting in an increasing number of cracks. For the internal friction angle, since the wear and fragmentation of particles are the main causes of its deterioration, it is mainly controlled by the flux of the change in water content, so it is significantly impacted by the cycle amplitude.

\subsection{Evolution of Loess Pore Cumulative Volume Distribution} under the Action of Dry-Wet Cycles. To further explore the differences in the evolution of the pore structure in the loess samples after several dry-wet cycles, NMR tests were performed on the samples at the optimal moisture content after the $0^{\text {th }}, 1^{\text {st }}, 3^{\text {rd }}$, and $5^{\text {th }}$ cycles, and different dry-wet conditions were obtained. The T2 spectrum curve under the number of cycles is shown in Figure 7.

Figure 7 shows one higher peak and two lower peaks, and the relaxation time at the peak position is concentrated between 1 and $10 \mathrm{~ms}$. After 0 dry-wet cycles, the curve is the lowest, and the distribution interval is the narrowest, indicating that the pores are the smallest at this time. With the increase in the number of dry-wet cycles, the peak of the T2 spectrum curve and the position of the higher peak clearly move upward. This wave peak exhibits slight fluctuations. In the $1^{\text {st }}$ and $3^{\text {rd }}$ dry-wet cycles, the curve peak shifts greatly, and the distribution becomes wider. The total integrated area under the T2 spectrum curve is the total pore volume. This relationship can be analyzed. With 1 to 3 wet-dry cycles, the pore changes are more obvious, and the pore changes then tend to become stable, indicating that the first three wet and dry cycles significantly change the pore structure of the loess, resulting in obvious degradation.

According to the principle of NMR, the $\mathrm{T} 2$ relaxation is related to the pore size of compacted ancient soil. Therefore, considering the structural characteristics of the test block, the surface relaxation strength is $3.0 \mu \mathrm{m} / \mathrm{s}$. Assuming that the pores in the test block are ideally spherical and $(\mathrm{S} / \mathrm{V})$ pore $=3 / r$, the relationship between pore radius and relaxation time is as follows:

$$
r=9.0 T_{2}
$$

For the sake of comparison, this study follows Lei [22] to classify and calibrate the microscopic pores of loess. The pore sizes are divided into four categories: large pores $(D>32 \mu \mathrm{m})$, medium pores $(8 \mu \mathrm{m} \leq D \leq 20 \mu \mathrm{m})$, small pores $(2 \mu \mathrm{m}) \leq D<8 \mu \mathrm{m})$, and micropores $(D<2 \mu \mathrm{m})$. According to the above formula, combined with the spectrum area, the percentages of each pore size range are calculated, and the $T_{2}$ spectrum relaxation time distribution of the sample can then be converted into the pore size distribution. According to the pore sizes, the pore size distribution map can be drawn. To more intuitively reflect the regular pore size change in the soil and the pore size distribution of the pores, the test soil pore sizes are divided into $(0,2),[2,8],(8,32]$, and $(32,6000]$ (unit: $\mu \mathrm{m}$, the same below), for a total of 4 groups. This calculation can obtain the average content distributions of various pore sizes inside the soil samples after different numbers of dry and wet cycles, as shown in Figure 8.

Figure 8 shows that, with increasing numbers of dry-wet cycles, the micropores gradually decrease, the small pores fluctuate irregularly, the medium-sized pores gradually increase, and the number of large pores is very low. The reason is that after the dry-wet cycles, the micropores and small pores gradually transform into small- and medium-sized pores. The effect of water in the humidification process causes the small particles on the surfaces of large particles to be lost with the flow of water, and the initial structure is damaged. The pores continue to evolve into small- and medium-sized pores, increasing the number of medium- 

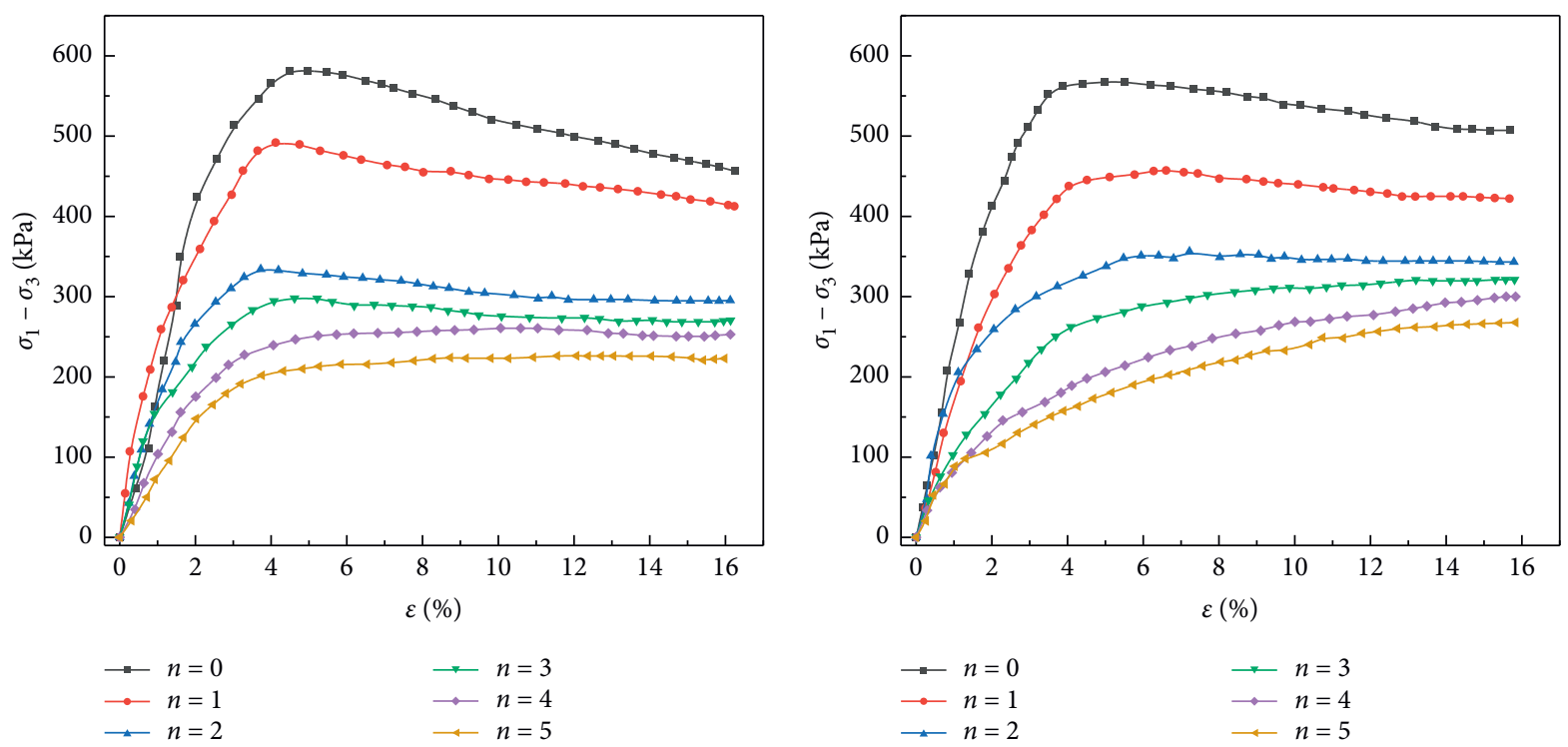

(a)

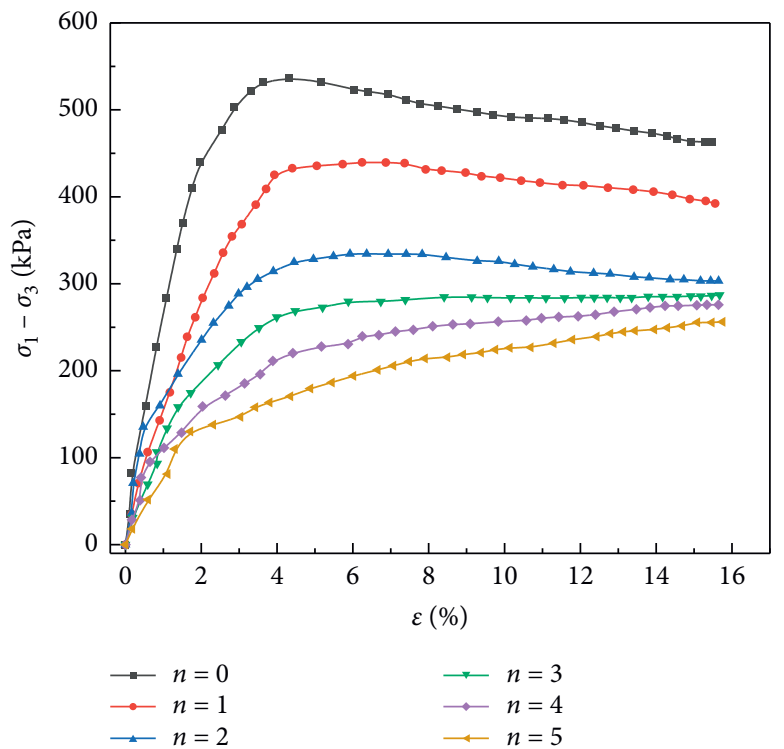

(c)

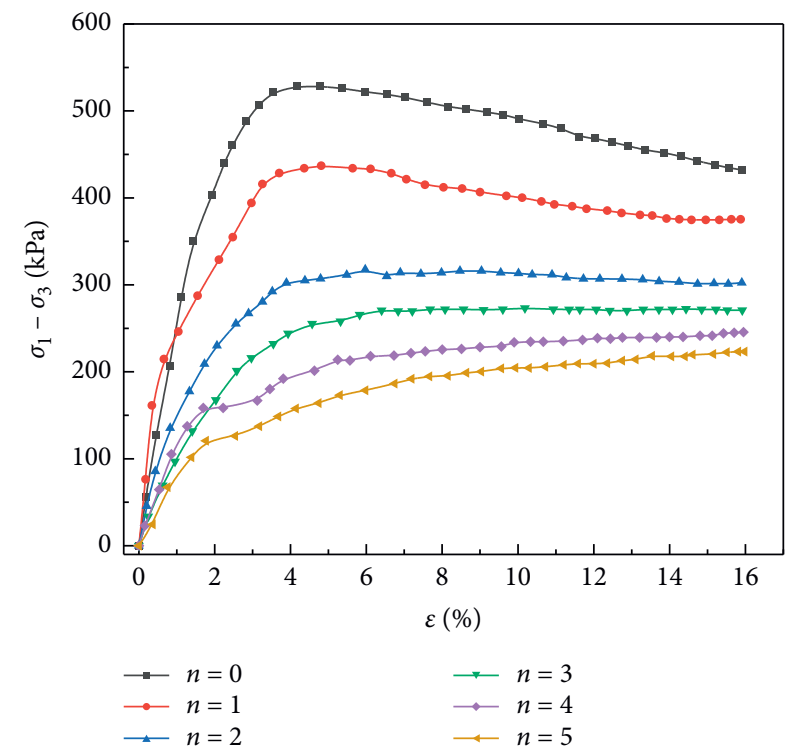

(d)

Figure 3: Stress-strain curves under different dry-wet cycle paths at a confining pressure of $100 \mathrm{kPa}$. (a) Dry and wet cycle path a. (b) Dry and wet cycle path b. (c) Dry and wet cycle path c. (d) Dry and wet cycle path d.

sized pores. In addition, as tiny particles and soluble salts on the surface of the pellets are washed away, the number of pores inside the pellets increases, and the total porosity increases. During the dehumidification process, the soil shrinks. Dry shrinkage develops cracks and microcracks in the soil, which increase the porosity of the compacted loess. Figure 7 shows that the spectrum area increases with increasing numbers of wet and dry cycles.

3.4. Granular Structure Effect under the Action of Dry-Wet Cycles. To analyze the changes in the particle structure of the loess samples after several cycles of drying and wetting, SEM experiments were performed on the samples at the optimal moisture content after $0,1,3$, and 5 cycles. This article presents results for only 0 and 5 times. Electron micrographs magnified 3000 times after the dry and wet cycles are shown in Figure 9.

The pores and cracks analysis system (PCAS) software is used for microscopic quantitative analysis of the particle pores and fracture images [23]. After the SEM image is vectorized by the software, the changes in the abundance, average shape factor, and fractal dimension of the shape distribution during the dry and wet cycles are analyzed to reveal the changes in the soil structure at the micro level.

3.4.1. Soil Abundance. The ratio of the short axis of a soil particle to its long axis is the pore abundance C [24], and the expression for soil particle abundance is 


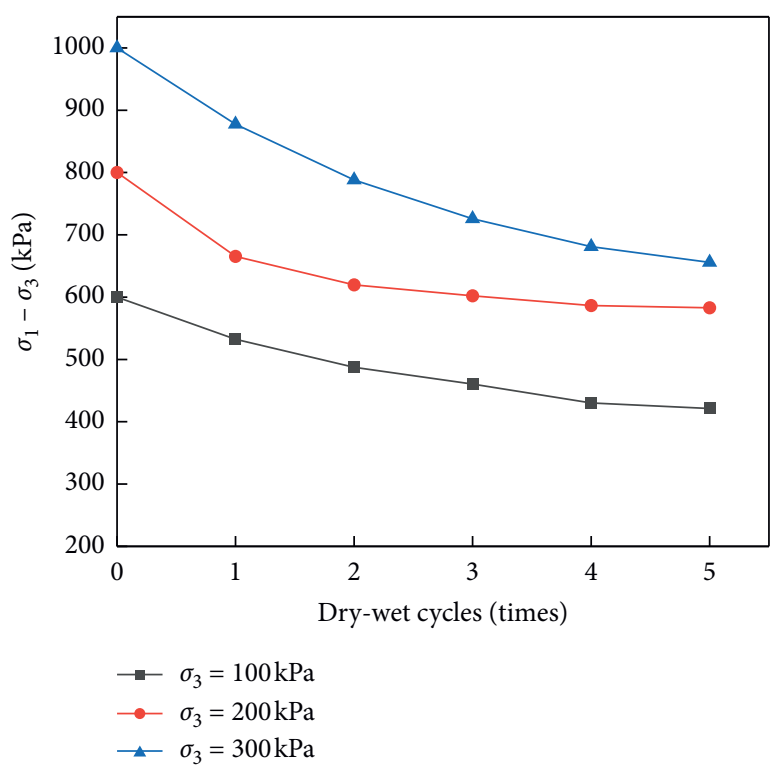

(a)

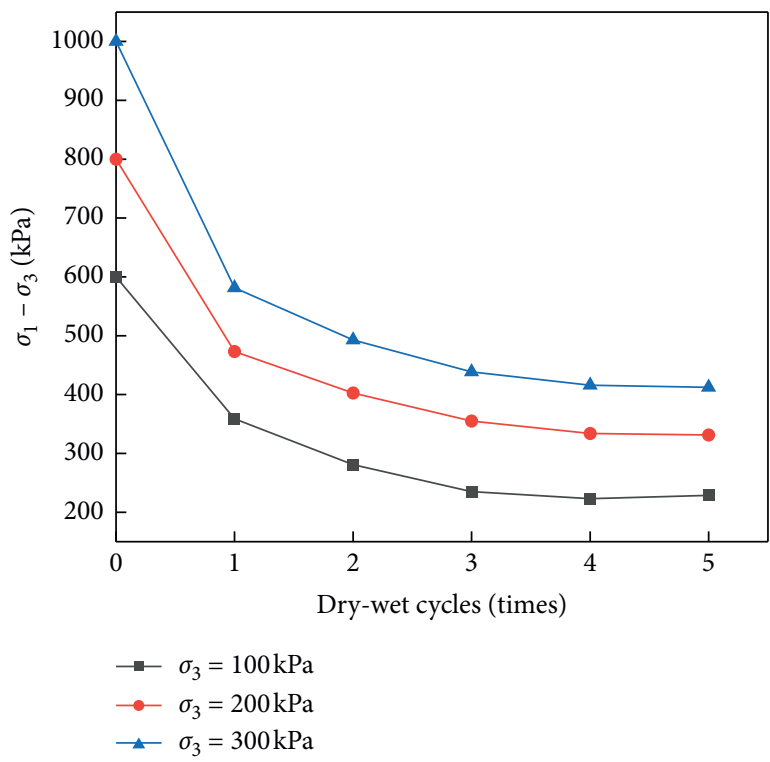

(c)

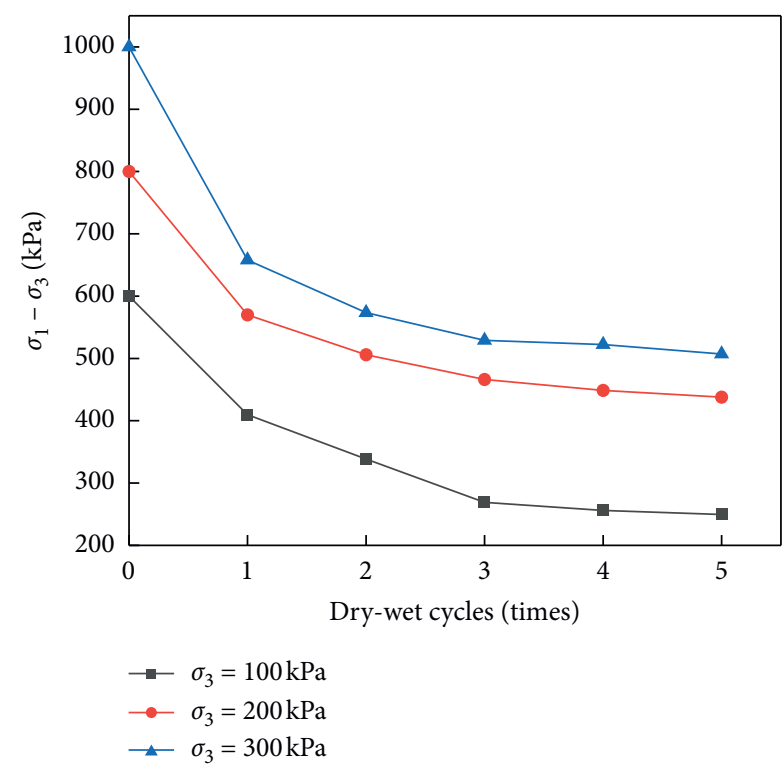

(b)

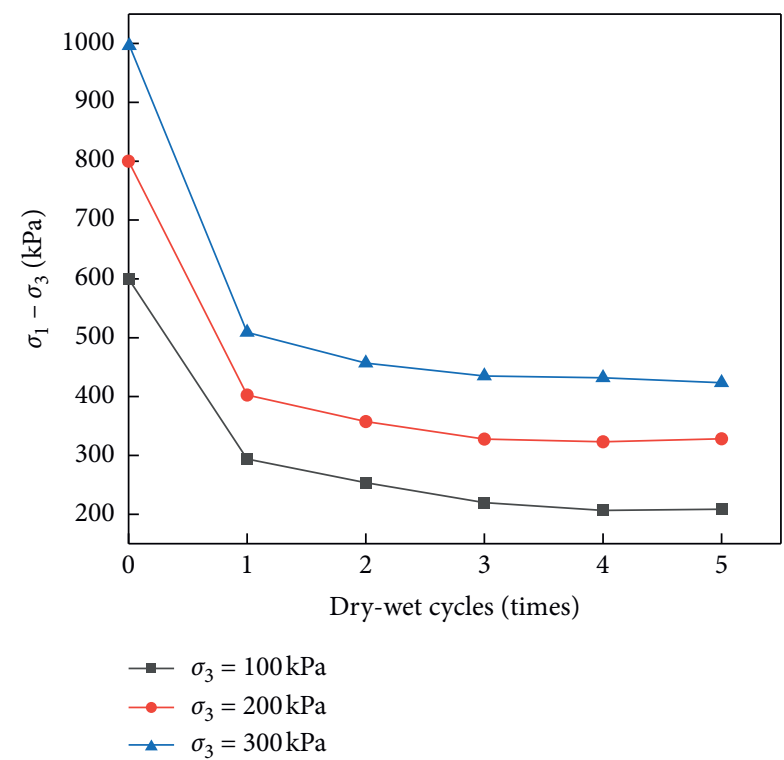

(d)

Figure 4: The relationships between the triaxial shear strength and the number of dry-wet cycles for different circulation paths. (a) Dry and wet cycle path a. (b) Dry and wet cycle path b. (c) Dry and wet cycle path c. (d) Dry and wet cycle path d.

$$
C=\frac{B}{L}
$$

In the formula, $C, B$, and $L$ represent the pore abundance, pore minor axis length, and pore major axis length, respectively, where the $C$ value ranges between 0 and 1 . The closer the $C$ value is to 1 , the more circular the pores are; otherwise, the pores tend to be elongated.

According to Figure 10, the distribution of pore abundance values before and after shearing in each group of pictures basically shows a normal distribution; the values are mainly concentrated between 0.4 and 0.9 , accounting for approximately $84.5 \%$ to $90.5 \%$ of the total. Among them, the distribution is mostly between 0.5 and 0.8 , accounting for approximately $65 \%$ to $70.4 \%$ of the total, which shows that the overall shape of the pores tends to be oblate and quasispherical, with few elongated and round pores. As the drywet cycles progress, the abundance gradually increases in the range from 0.4 to 0.7 , and the other values generally show a decreasing trend, indicating that the dry-wet cycles increase the roundness of soil particles through the effect of water on the edges and corners of the particles. The obvious scouring effect, combined with the friction between particles, promotes changes in the abundance of particle units.

3.4.2. Average Shape Factor of Microscopic Pores of Soil Samples. The average shape factor [25] is denoted by F: 


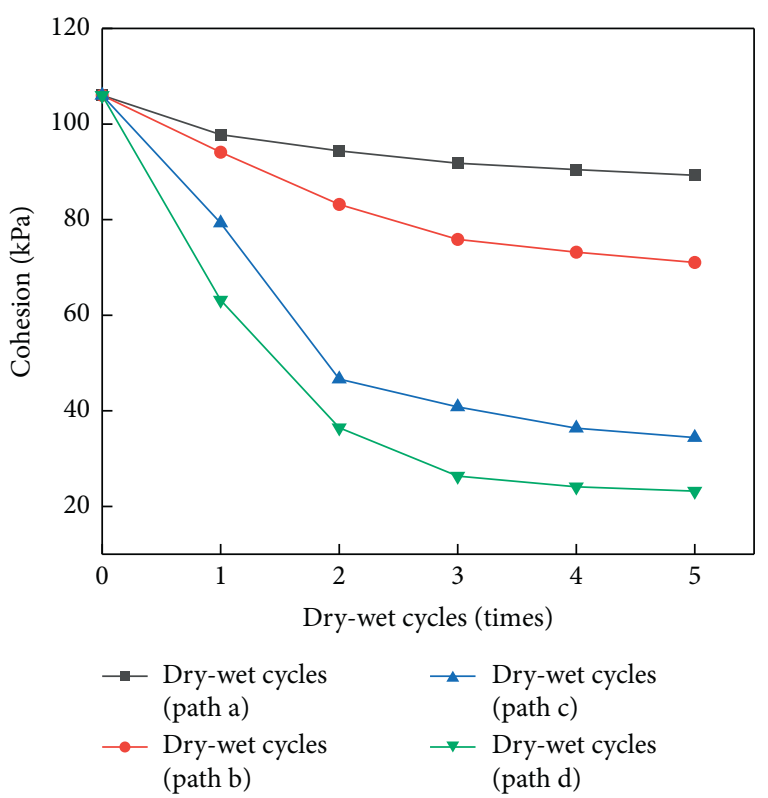

Figure 5: The relationship between wet and dry cycles and cohesion.

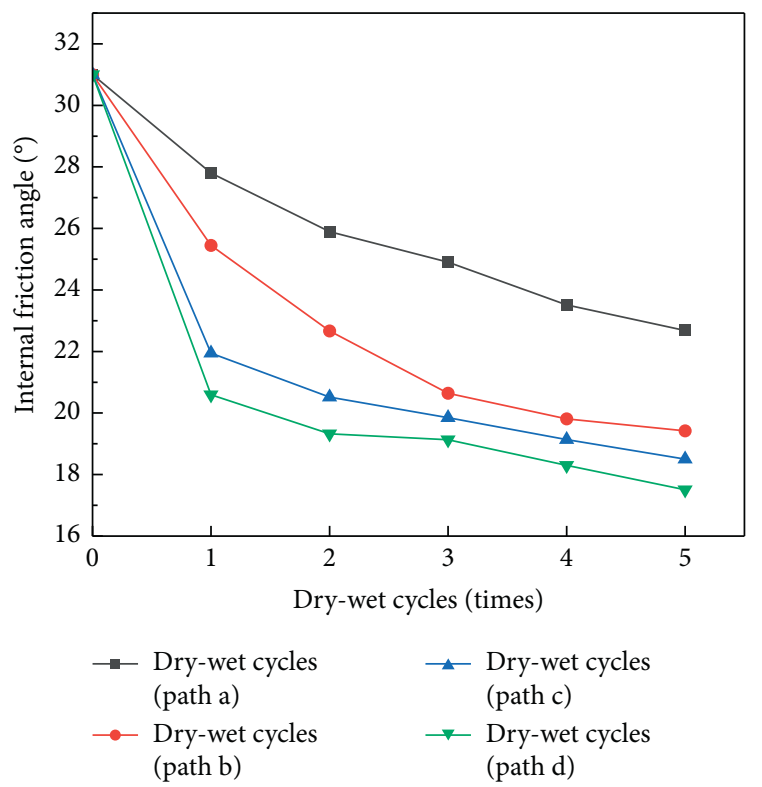

FIgURE 6: The relationship between the number of wet and dry cycles and the angle of internal friction.

$$
F=\frac{\sum_{i=1}^{n} F_{i}}{n}
$$

In the formula, " $F$ " is the pore morphology coefficient, " $P$ " is the circumference of a circle with the same area as the pore, and " $S$ " is the actual circumference of the pore.

Figure 11 shows the changes in the average shape coefficient after different dry-wet cycles. The figure illustrates that, with increasing numbers of dry-wet cycles, the average shape coefficient curve continuously decreases; the decrease is greater before 3 cycles, and the curve tends to stabilize

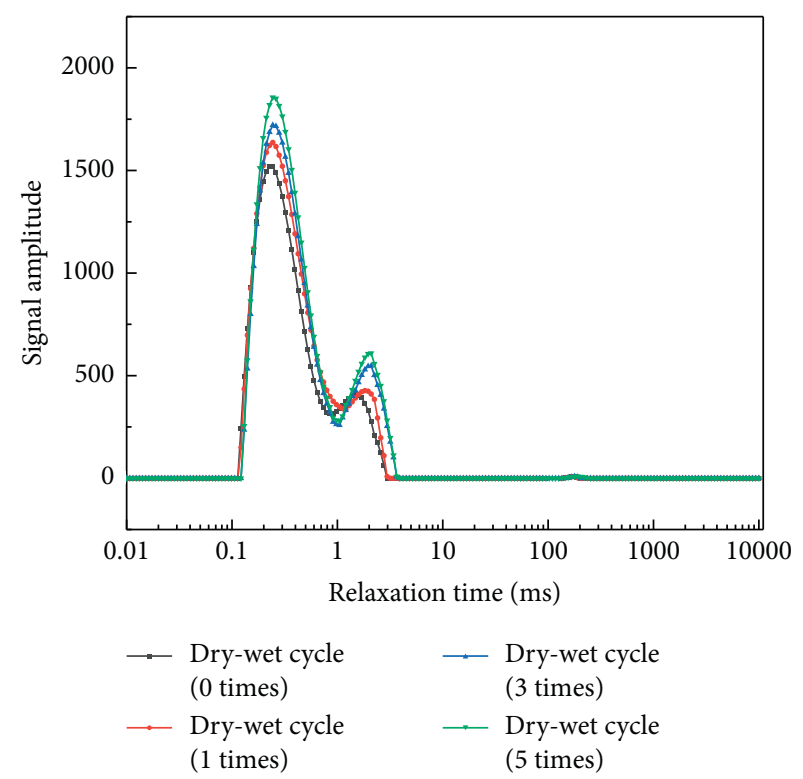

FIGURE 7: T2 spectrum distribution under different dry-wet cycles.

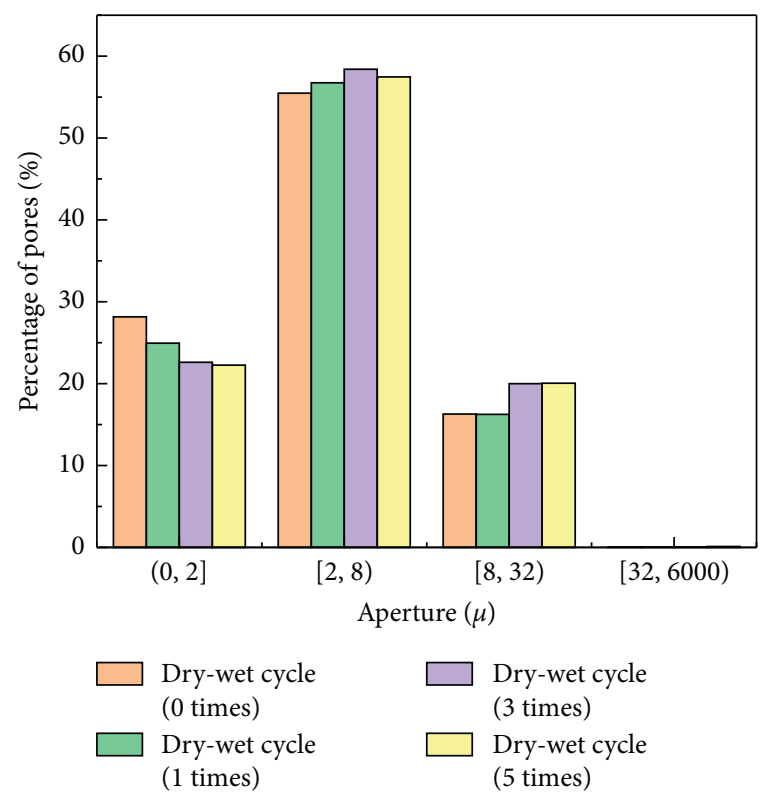

Figure 8: Pore content distribution of each group.

after 3 cycles. The reason why 0 to 3 cycles have great impacts on the original structure of the soil is analyzed. The structures and shapes of the soil particles change greatly, and the roundness of the soil increases greatly. The original aggregate structure of the soil is also destroyed, and damage occurs. The attached debris begins to leave the aggregates, causing the soil particle structure to become chaotic. After 3 cycles, the structural damage tends to be stable, and the shape coefficient changes a little.

3.4.3. Change in Fractal Dimension of Morphological Distribution. The linear relationship between the equivalent 


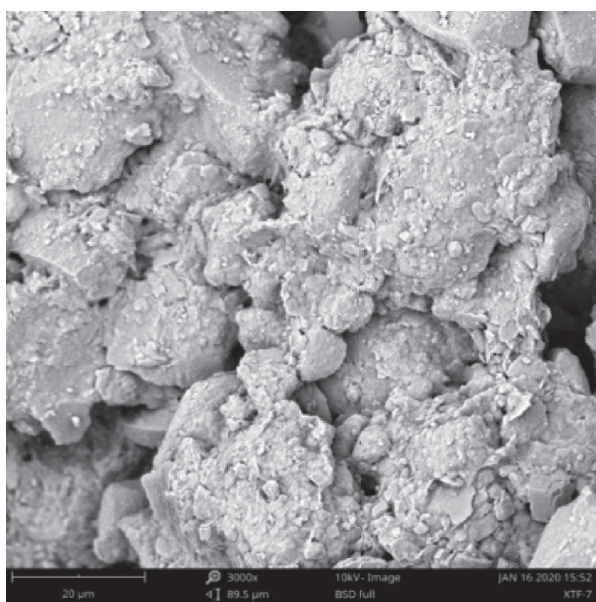

(a)

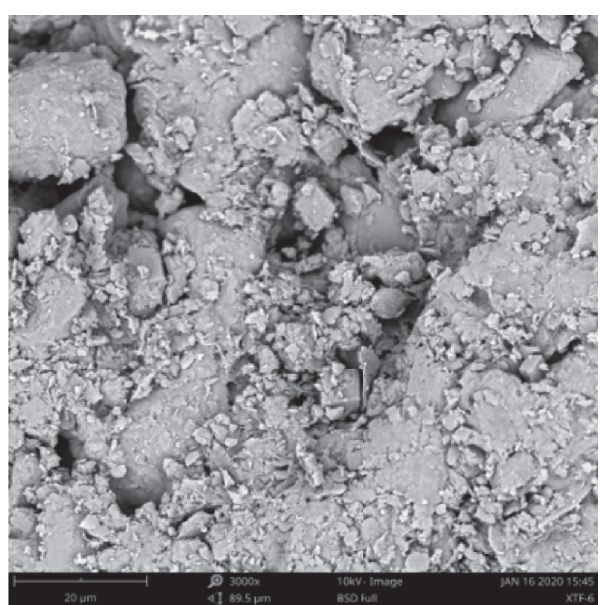

(b)

Figure 9: SEM images after different numbers of wet and dry cycles: (a) 0 cycles and (b) 5 cycles.

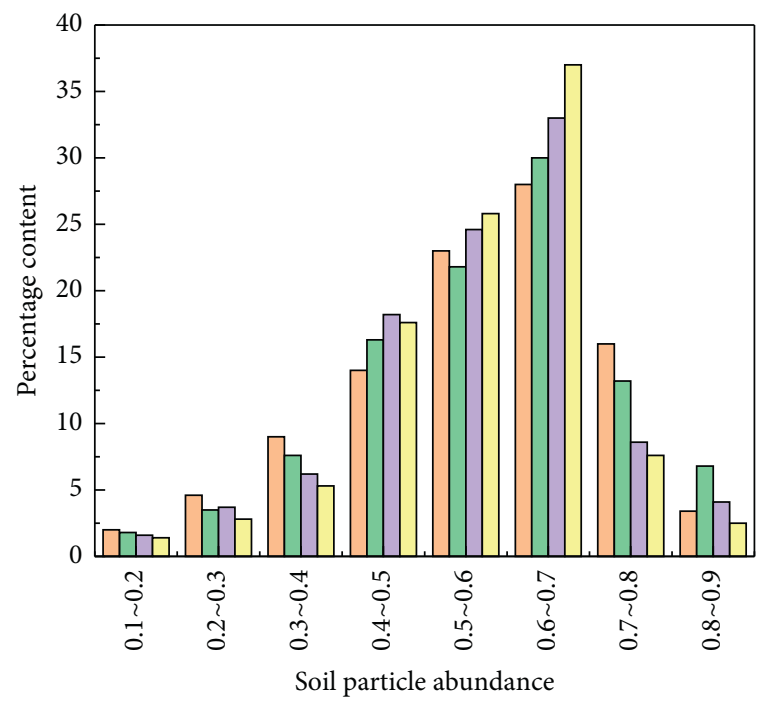

Dry-wet cycle (0 times) $\square$ Dry-wet cycle (3 times) Dry-wet cycle (1 times) Dry-wet cycle (5 times)

FIGURE 10: Variations in soil particle abundance under the action of dry-wet cycles.

area and the perimeter of the fractal dimension of the shape distribution is calculated:

$$
\log (\text { Perimeter })=\frac{D}{2} \times \log (\text { Area })+C .
$$

In the formula, Perimeter is the equivalent perimeter of the pore, Area is the equivalent area of the pore, " $\mathrm{D}$ " is the fractal dimension of the pore shape distribution, and " $\mathrm{C}$ " is the fitting constant.

Figure 12 is a graph showing the relationship between the number of dry-wet cycles and the fractal dimension.

The figure reveals that the fractal dimension decreases with increasing numbers of dry and wet cycles; the decrease is the largest after the first cycle, and the decreasing trend tends to become stable after the third cycle. This relationship

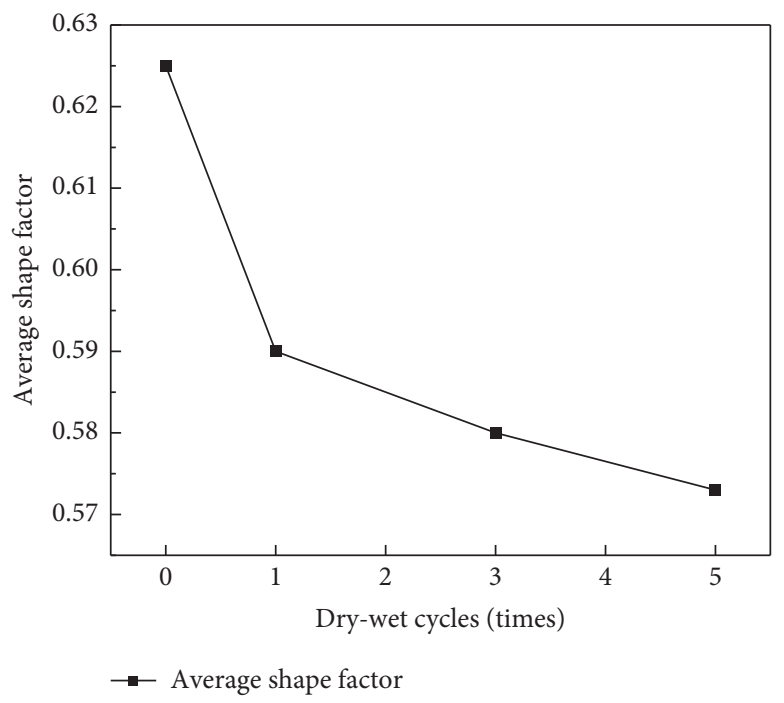

FIGURE 11: Change in the average shape coefficient after different wet and dry cycles.

implies that, during the dry-wet cycles of the unearthed samples, the connections between soil particles are broken due to the dry-wet cycles, and the cementation is weakened. The original cemented structure is continuously destroyed, decomposed, and polished to form irregular particles. Thus, the particle fractal dimension changes.

\section{Multiscale Mechanism Analysis of Loess Collapse under the Action of Dry-Wet Cycles}

The scale effect means that a certain spatial structural characteristic can be expressed only at a certain sampling scale. Different research scales can determine the strength degradation of loess under different numbers of dry-wet cycles. On the microscopic scale, the pores of loess samples that have not been subjected to dry-wet cycles are small and unevenly distributed. The minerals are connected directly and firmly. After the dry-wet cycles, the connections 


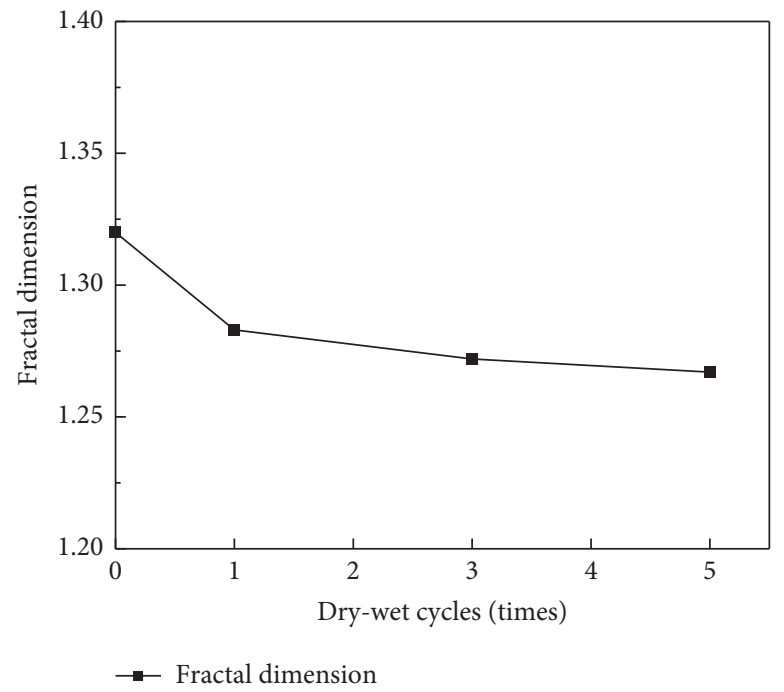

FIGURE 12: The relationship between the number of wet and dry cycles and the fractal dimension.

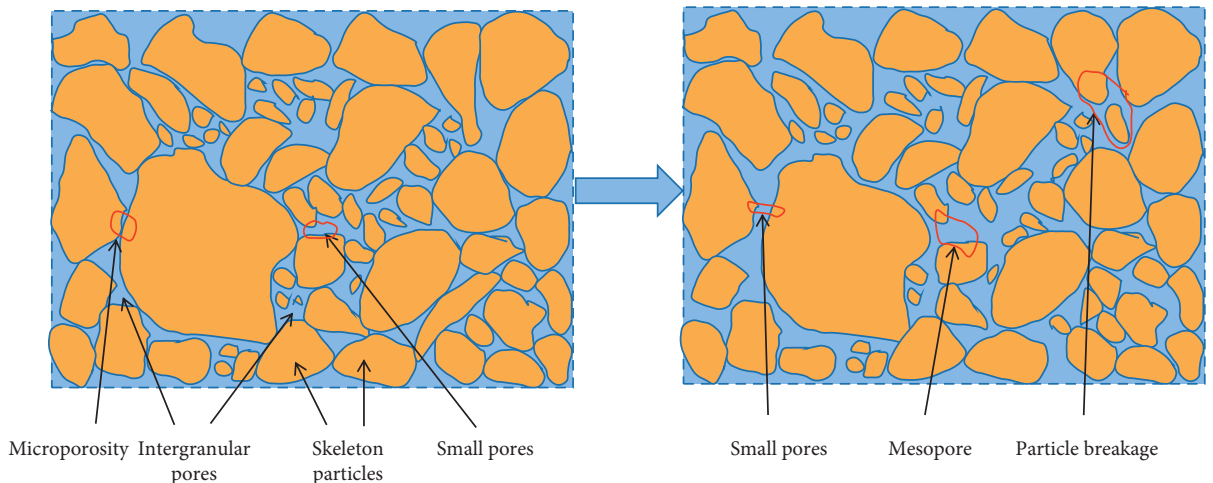

Figure 13: Schematic diagram of the fine microstructure of loess before and after dry and wet cycles.

between loess particles gradually change from surface-tosurface contacts and surface-to-edge contacts to edge-toedge contacts and point-to-edge contacts. On the microscopic scale, according to the NMR analysis, the pores and micropores of the samples are transformed into small pores and small pores into medium-sized pores after the sample undergoes wet-drying cycles. The overall porosity increases, indicating that the strength of the soil is degraded and attenuated under the action of the dry-wet cycles; the macroscopic triaxial tests can directly show that the cohesive force and internal friction angle of the loess after the dry-wet cycles decrease, and the intensity of shear resistance is reduced. From the micro-, meso-, and macroscales, the internal structure of the loess is damaged and deteriorates under the action of dry and wet cycles.

The changes in soil particles, pores, and clots before and after the dry-wet cycles are drawn as a schematic diagram in Figure 13. The liquid bridges between soil particles break, resulting in cracks in the soil; on the other hand, as the surfaces of the aggregates are washed clear of tiny particles and soluble salts, the number of pores inside the aggregates increases, and the total porosity increases. During the dehumidification process, the soil shrinks. Dry shrinkage develops cracks and microcracks in the soil, which increases the porosity of the compacted loess. Under the action of the dry-wet cycles, the tiny pores gradually turn into small- and medium-sized pores, the soil particles are partially broken under the action of water, and the overall porosity increases, changing the loess structure, which in turn reduces the macroscopic strength of the loess.

Under the action of water, the physical and mechanical properties of loess undergo remarkable changes, but the generation of cracks in the loess body is a precondition for these changes. Loess slope construction generally occurs in the dry season. The surface of the newly excavated slope is affected by weather conditions such as wind and sun. The water on the surface of the slope continuously evaporates, and the water inside the slope continuously migrates to the surface of the slope. The moisture within a certain range of the slope surface continues to decrease, and the matrix suction of the soil continually increases, causing the soil to shrink and deform. When the generated stress is greater than the strength of the soil, cracks form inside the soil. The generation of loess cracks involves physical and 
chemical interactions between water and soil. Due to the presence of strong hydrophilic clay minerals such as montmorillonite, illite, and kaolinite in loess and given the effects of collapsibility and shrinkage during water loss, rifts form. The formation of vertical joints in loess is mainly due to the uneven thickness of the loess that reflects undulating topography and to the uneven collapse and deformation under the action of water, which leads to the generation of internal joints. As the loess continues to accumulate, when the top pressure increases, the soil at the top of the joint fissure is stressed. When the strength of the soil is reached, the inner joint fissure expands in the vertical direction to form a vertical joint. Under the action of water, the slope gradually becomes saturated from the surface to the interior of the slope. Under the action of the water wedge pressure, the original fissures open, expand, and deepen. When the moisture content in loess increases, the thickness of the combined water increases, and the distance between the clay particles increases; the van der Waals force, the water glue connection and the capillary force between the particles decrease, while the water-resistant cement softens and the connection strength decreases. When the moisture content of the soil sample increases during a dry-wet cycle, the volume of bound water and gravitational water entering the loess clay particles also increases, and the distance between the clay particles wedged by water increases, which leads to an increase in the water content between the particles. The faster the force decreases, the greater the softening degree of the water-resistant cement, and the greater the reduction in the friction coefficient between grains and the friction between grains; these changes lead to an increase in the shear strength of the loess sample. When the hydrostatic pressure and hydrodynamic pressure generated by the humidification process are greater than the tensile strength of the soil, on the one hand, new cracks are generated within the soil; on the other hand, these cracks are connected to each other, resulting in destruction of the integrity and uniformity of the soil. At the same time, the cohesive force between soil particles is weakened, resulting in structural damage and reduced soil strength. The Luochuan loess has high contents of clay particles, mainly montmorillonite, illite, and kaolinite, and these clay minerals disintegrate. The greater the clay content, the greater the water absorption of the soil, the greater the moisture change, and the greater the disintegration. During the humidification process, the easily disintegrated particles of the loess clay minerals swell, disintegrate, and break. Due to the uneven distribution of mineral components, structures, and water aggregates in the loess body, the generation of microcracks is aggravated, and such microcracks inevitably cause stress concentrations in the soil and further accelerate the development and connection of cracks. In this way, the humidification and dehumidification processes near the surface of the slope continue to cyclically change, the shear strength of the slope continuously decreases, and the cracks in the soil continue to expand. When a certain dominant surface in the soil is penetrated, the loess slope collapses.

\section{Conclusions}

(1) After many triaxial tests with different numbers of dry and wet cycles, the stress-strain curve hardening phenomenon is obvious. Under the action of the first 3 dry-wet cycles, the cohesion and the angle of internal friction decrease sharply, and the shear strength of the loess deteriorates significantly; the loess stabilizes after 3 cycles. Given different circulation paths, for circulation path $d$ (large circulation amplitude), the shear strength of the soil is reduced by $51 \%$, and the strength deterioration of the loess is the most significant.

(2) On the mesoscale, using nuclear magnetic resonance analysis, during the processes of drying and wetting, the tiny particles on the surfaces of soil particles are lost with the flow of water; the initial structure is damaged, and the micro- and small pores evolve to small- and medium-sized pores. The number of medium-sized pores increases; in addition, as the tiny particles and soluble salts on the surfaces of the pellets are washed away, the number of pores inside the pellets increases, and the total porosity increases.

(3) Microscopically, using scanning electron microscopy, with increasing numbers of dry and wet cycles, the internal structural units of loess change significantly, and the particle connections weaken. The connections gradually change from surface-to-surface contacts and surface-to-edge contacts to edge-to-edge contacts and edge-to-point contacts. The integrity of the loess continues to decrease, and internal passages continue to develop.

(4) After multiscale analysis of the loess samples, the generation of loess cracks can be preliminarily determined to involve physical and chemical interactions between water and soil. Due to the presence of strong hydrophilic clay minerals such as montmorillonite, illite, and kaolinite in the loess, cracks are formed under the effects of water soaking and shrinking. The development of vertical joints in loess is mainly due to the uneven thickness of the loess because of the undulating topography and to the uneven collapse and deformation under the action of water, which leads to the generation of internal joints.

\section{Data Availability}

The data used to support the findings of this study are available from the corresponding author upon request.

\section{Conflicts of Interest}

The authors declare that they have no conflicts of interest.

\section{Acknowledgments}

The authors gratefully acknowledge the financial support from the National Natural Science Foundation of China 
(grant no. 42072319) and the Key Science and Technology Program of Shaanxi Province (grant no. 2017ZDXM-SF082).

\section{References}

[1] D. Sh. Liu, Loess and the Environment, Science Press, Beijing, China, 1985.

[2] P. D. Qiao and Z. J. Li, Engineering Geology in Loess Area, China Water Conservancy and Hydropower Press, Beijing, China, 1994.

[3] R. M. Ma, Ch. F. Cai, Zh. X. Li et al., "Evaluation of soil aggregate microstructure and stability under wetting and drying cycles in two Ultisols using synchrotron-based X-ray micro-computed tomography," Soil and Tillage Research, vol. 149, pp. 1-11, 2015, ISSN 0167-1987.

[4] S. S. Razouki and M. Bushra, "Salem, Impact of soaking-drying cycles on gypsum sand roadbed soil," Transportation Geotechnics, vol. 2, pp. 78-85, 2015, ISSN 2214-3912.

[5] A. Pasculli, N. Sciarra, L. Esposito, and A. W. Esposito, "Effects of wetting and drying cycles on mechanical properties of pyroclastic soils," Catena, vol. 156, pp. 113-123, 2017, ISSN 0341-8162.

[6] Y. K. Wu, K. J. Shi, Y. Han, T. Han, J. L. Yu, and D. D. Li, "Experimental study on strength characteristics of expansive soil improved by steel slag powder and cement under dry-wet cycles," Iranian Journal of Science and Technology, Transactions of Civil Engineering, 2020.

[7] J. A. T. de Oliveira, A. Fabio, M. Cássaro, and L. F. Pires, "Estimating soil porosity and pore size distribution changes due to wetting-drying cycles by morphometric image analysis," Soil and Tillage Research, vol. 205, 2021 ISSN 0167-1987, Article ID 104814.

[8] K. Liu, W. J. Ye, H. J. Gao, and Q. Dong, "Multi-scale effects of mechanical property degradation of expansive soils under drying-wetting environments," Chinese Journal of Rock Mechanics and Engineering, vol. 39, no. 10, pp. 2148-2159, 2020.

[9] W. J. Ye, Y. Q. Chen, D. F. Zhang, and Y. Bai, "Macro and micro experimental study on the influence of moisture migration on the strength of compacted loess under freezethaw," China Journal of Highway and Transport, vol. 1-12 http://kns.cnki.net/kcms/detail/61.1313.U.20200929.1323. 004.html.

[10] Y. L. Chou, S. S. Jia, Q. H. Zhang, W. Cao, and Y. Sheng, "The influence of freeze-thaw action on loess collapsibility coefficient considering soil structure," Rock and Soil Mechanics, vol. 39, no. 8, pp. 2715-2731, 2018.

[11] L. J. Su, Q. Zhao, H. Liu, W. J. Qian, J. T. He, and J. X. Yang, "Swelling and shrinkage behaviors and evolution law of crack morphology of undisturbed loess during wetting-drying cycles," Journal of Tianjin University (Science and Technology), vol. 54, no. 3, pp. 255-267, 2021.

[12] Ch. M. Hu, Y. L. Yuan, X. Y. Wang, Y. Mei, and Zh. Liu, "Experimental study on strength deterioration model of compacted loess under wetting-drying cycles," Chinese Journal of Rock Mechanics and Engineering, vol. 37, no. 12, pp. 2804-2818, 2018.

[13] L. Li, K. Zhang, Q. L. Zhang et al., "Experimental study on the loess strength degradation characteristics under the action of dry-wet and freeze- thaw cycles," Journal of Glaciology and Geocryology, vol. 38, no. 4, pp. 1142-1149, 2016.

[14] Sh. J. Wen, W. Y. Zhang, and C. Q. Zeng, "Experimental study on dynamic shear modulus and damping ratio of undisturbed loess in Haidong area," Chinese Journal of Geotechnical Engineering, vol. 41, no. S2, pp. 137-140, 2019.

[15] Z. L. Zhang, S. R. Wu, H. M. Tang, T. Wang, and P. Xing, "Dynamic characteristics and microcosmic damage effect of loess and mudstone," Chinese Journal of Rock Mechanics and Engineering, vol. 36, no. 5, pp. 1256-1268, 2017.

[16] J. Zhang, J. J. Zheng, W. Z. Cao, and H. J. Lai, "Dynamic characteristics of saturated compacted loess under cyclic loads," Chinese Journal of Geotechnical Engineering, vol. 35, no. S1, pp. 322-327, 2013.

[17] L. S. Tang and S. J. Wang, "Discussion on the mechanism and quantitative method of rock water chemical damage[J]," Chinese Journal of Rock Mechanics and Engineering, vol. 21, no. 3, pp. 314-319, 2002.

[18] J. Wang, P. Cao, Y. L. Zhao, and H. B. Chai, "Influence of chemical action of water-soil on soil shear strength[J]," Journal of Central South University (Science and Technology), vol. 41, no. 1, pp. 245-250, 2010.

[19] R. T. Yan, Z. T. Zeng, W. Q. Xu, Z. T. Zeng, H. Miao, and C. F. Wei, "Research progress on the physical and mechanical properties of clay under the variation of hydrochemical environment," Journal of Yangtze River Scientific Research Institute, vol. 31, no. 6, pp. 41-47, 2014.

[20] W. J. Ye, Y. Bai, C. Y. Cui, and X. Duan, "Deterioration of the internal structure of loess under dry-wet cycles," Advances in Civil Engineering, vol. 2020, Article ID 8881423, 17 pages, 2020.

[21] S. H. Zheng, J. L. Jin, H. L. Yao, and X. R. Ge, "Analysis of initial cracking behavior of expansive soil due to evaporation," Rock and Soil Mechanics, vol. 27, no. 12, pp. 2229-2233, 2006.

[22] X. Y. Lei, "Pore distribution characteristics of longdong loess in northern Shaanxi," Chinese Science Bulletin, no. 3, pp. 206-209, 1985.

[23] C. Liu, B. Shi, J. Zhou, and C. Tang, "Quantification and characterization of microporosity by image processing, geometric measurement and statistical methods: application on SEM images of clay materials," Applied Clay Science, vol. 54, no. 1, pp. 97-106, 2011.

[24] R. L. Hu, "Quantitative research progress of clay microstructure," in Proceedings of the Fifth National Engineering Geology Conference, vol. 6, Engineering Geology Committee of Chinese Geological Society, Hui County, China, 1996.

[25] Q. S. Meng, C. Yang, X. Z. Xu, X. W. Lei, and S. L. Sui, "Analysis of microstructure of soft clay before and after its improvement with dynamic consolidation by drainage," Rock and Soil Mechanics, no. 7, pp. 1759-1763, 2008. 